\title{
Direct-acting antivirals response in hepatocellular carcinoma: Does the presence of hepatocellular carcinoma matter?
}

\author{
Chung-Feng Huang ${ }^{1,2,3}$ and Ming-Lung Yu, ${ }^{1,2,4,5}$ \\ 'Hepatobiliary Division, Department of Internal Medicine, Kaohsiung Medical University Hospital, Kaohsiung Medical University, \\ Kaohsiung: ${ }^{2}$ Faculty of Internal Medicine and Hepatitis Research Center, College of Medicine, and Center for Lipid Science and Aging \\ Research Center, Kaohsiung Medical University, Kaohsiung; ${ }^{3}$ Department of Occupational Medicine, Kaohsiung Medical University \\ Hospital, Kaohsiung Medical University, Kaohsiung; ${ }^{4}$ Institute of Biomedical Sciences, National Sun Yat-Sen University, Kaohsiung: \\ ${ }^{5}$ Center For Intelligent Drug Systems and Smart Bio-devices (IDS ${ }^{2} B$ ) and Department of Biological Science and Technology, College of \\ Biological Science and Technology, National Chiao Tung University, Hsin-Chu, Taiwan
}

During the clinical trial development of directly acting antivirals (DAAs), evidence regarding the treatment efficacy in chronic hepatitis $\mathrm{C}$ patients with hepatocellular carcinoma (HCC) was scarce because these patients have always been excluded. Apart from the clinical trials, more HCC patients are currently being treated in daily practice, given that these treatments are highly effective and involve well-tolerated regimens. Large scale, real-world studies have demonstrated potentially suboptimal antiviral treatment efficacy in HCC patients who received DAAs. It is postulated that the impairment of the bioavailability of DAAs may account for the inferior treatment response. However, the results could not be generalized across all studies. The differing results were attributed to diverse patient characteristics, suboptimal regimens or imprecise definitions of active cancer statuses at the time of treatment initiation. Additional large-scale studies that utilize the treatment of choice in clearly defined HCC patients with different disease severities are warranted to clarify the issue. (Clin Mol Hepatol 2019;25:168-171)

Keywords: Hepatocellular carcinoma (HCC); Chronic hepatitis C (CHC); Hepatitis C virus (HCV)

\section{INTRODUCTION}

Liver cancer, mainly hepatocellular carcinoma (HCC), is the second most frequent cause of cancer-related death worldwide. ${ }^{1,2}$ Hepatitis C virus (HCV) is one of the major etiologies of HCC and is attributed to approximately $31 \%$ of HCC cases worldwide. ${ }^{1} \mathrm{HCV}$ eradication by the use of antiviral therapy greatly reduces HCC risks. ${ }^{3,4}$ Conversely, the question arises as to whether the presence of HCC affects the efficacy of antiviral treatment. Due to the limited sustained virological response (SVR) rate and the unfavorable safety concerns to cancer patients, this issue has not received significant attention in the interferon era. In addition, due to the arduous and prolonged treatment course and the uncertain longterm benefits, cancer patients who were enrolled in interferonbased studies were always classified in the curative HCC status before the initiation of treatment. Huang et al. have treated 82 consecutive chronic hepatitis C (CHC) patients with curative HCC status and 87 sex/age-matched, cirrhotic, non-HCC controls by

\section{Abbreviations:}

CHC, chronic hepatitis C; DAAs, direct-acting antiviral agents; HCC, hepatocellular carcinoma; HCV, hepatitis C virus; SVR, sustained virological response

\section{Corresponding author: Ming-Lung Yu}

Hepatobiliary Division, Department of Internal Medicine, Kaohsiung Medical University Hospital, No.100, Tzyou 1st Road Kaohsiung 807, Taiwan

Tel: +886-7-312-1101 ext. 7475, Fax: +886-7-312-3955

E-mail:fish6069@gmail.com

https://orcid.org/0000-0001-8145-1900 
the use of pegylated interferon in combination with weight-based ribavirin. ${ }^{5}$ The SVR rate was significantly lower in the HCC group compared to the non-HCC group $(48.8 \%$ vs. $64.4 \%, P=0.04)$. However, the inferior treatment result in the HCC group was observed among the genotype-1 patients (33.3\% vs. $60.7 \%$, $P=0.005$ ) but was not observed ingenotype-2/3 infected patients $(70.6 \%$ vs. $71.0 \%, P=0.88) .{ }^{5}$ Since 2014 , all oral, directly acting antivirals (DAAs) have replaced interferon-based therapy as the standard treatment of CHC. This standard of care opens new horizons for $\mathrm{CHC}$ patients with more advanced liver disease, including hepatic decompensation and active HCC. However, the question arises as to whether the presence of HCC affects the antiviral potency in the current era of DAAs treatment.

\section{PILOT STUDY IN THE DAA ERA}

Evidence regarding the treatment efficacy of DAAs for $\mathrm{CHC}$ patients with HCC was scarce during clinical trial development because these patients were always been excluded. A rare exception was a phase 2 study, which included 61 HCC patients who were on the waiting lists for liver transplantation. Of all the patients who were allocated to sofosbuvir and ribavirin regimen, only $49 \%$ had undetectable post-transplantation HCV RNA 12 weeks after the end of the treatment (SVR12). While participants were restricted to only those patients who had undetectable HCV RNA at the time of liver transplantation, $30(70 \%)$ of the 43 patients achieved SVR12 post-transplantation. ${ }^{6}$

\section{STUDIES INDICATING TREATMENT INFERIOR- ITY IN HCC PATIENTS}

Aside from the clinical trials, more HCC patients are currently being treated in daily practice, given that the regimens are with short duration, highly effective and well-tolerated. Saberi et al. first reported a SVR12 rate of 21 active HCC patients before liver transplantation, of which 7 patients (33.3\%) relapsed from various DAA regimens following the cessation of treatment. ${ }^{7}$ Additionally, Soria et al. reported another case series study regarding the treatment efficacy of DAA in HCC patients. ${ }^{8}$ Coincidently, they observed that all 5 patients with active HCC experienced virological relapses, compared to 12 relapses reported in the non-HCC patients (relapse rate: $100 \%$ vs. $8 \%$, respectively, $P<0.0001$ ). ${ }^{8} \mathrm{Al}-$ though these two preliminary reports were limited by the small case numbers, the diverse patient characteristics and certain suboptimal regimens, it delivered the signal for further elucidation upon this issue. Two recent large-scale studies have validated these preliminary observations. Prenner et al. enrolled 421 cirrhotic $\mathrm{CHC}$ patients, one-third of whom had a history of active HCC. ${ }^{9}$ The overall treatment failure rate was $21 \%$ in the HCC patients, compared to a treatment failure rate of only $12 \%$ in those patients without HCC $(P=0.01)$. Furthermore, of the 64 patients who had viable tumors at the time of DAA treatment, 27 patients (43\%) failed to achieve SVR12. Conversely, of the 71 patients who had previous HCC but were successfully treated with either surgical resection or transplantation, only 2 patients (3\%) failed HCV therapy. The treatment response of the patients with curative $\mathrm{HCC}$ was identical to those without HCC history. After adjusting for potential confounding factors, including treatment regimens, HCV genotypes and liver function reserves, the authors identified that patients with active $\mathrm{HCC}$ had an eight-fold increased risk of failing HCV therapy compared to patients without HCC. ${ }^{9}$ Another report examined the real world experiences of 15,884 CHC patients in the Veterans Affairs Health Care System in the United States. The overall SVR rates were substantially higher in the non-HCC group $(91.1 \%)$ and in the HCC post-liver transplantation group $(94.0 \%)$ than in the HCC group (74.4\%). The HCC patients were less likely to achieve SVR than the non-HCC patients (adjusted odds ratio: 0.38 [95\% confidence intervals $0.26-0.45]$ ), after adjusting for $\mathrm{HCV}$ genotype, cirrhosis, and other confounding factors. Although the study was limited by failing to define cancer curative status precisely at the time of DAA treatment, the big data provide a wide view regarding the potency of DAA in this special population. ${ }^{10}$ Taken together, these studies demonstrate an excellent SVR rate after liver transplantation $n^{9,10}$ but rather high pre-transplantation virological failure rate, ${ }^{6,7}$ while also raising concerns for clinicians in deferring the use of antiviral therapy for HCC patients on the transplantation list, particularly in areas where donors and facilities are at a high volume.

\section{POSTULATED MECHANISMS OF SUBOPTI- MAL ANTIVIRAL ACTIVITY IN HCC PATIENTS}

There are several proposed mechanisms for attenuated antiviral activity in HCC patients: First, HCCs are partially supplied by the branches of the hepatic arteries. DAA may have a suboptimal drug delivery via the portal systems. Second, the subverted cellular architecture of HCC foci may impair the bioavailability of 
DAAs, with these foci providing sanctuary sites for HCV replication." The theory of a viral replicating reservoir may be partially explained by a similar end-of-treatment response but higher relapse rate, as noted by Prenner et al. ${ }^{9}$ Third, HCC may down-regulate certain carrier or membrane proteins, which may then impair DAA uptake. ${ }^{12,13}$ Finally, HCC patients are prone to having impaired innate and adaptive immune responses, ${ }^{14}$ which may then have a negative impact on viral clearance during DAA treatment. ${ }^{15}$

\section{STUDIES FAVORING EQUAL TREATMENT EF- FICACY IN HCC PATIENTS}

Of 110 Asian Americans with sofosbuvir-based DAAs, Chang et al. observed an SVR rate of $82 \%$ (14/17) in HCC patients, which did not statistically differ from those without HCC $(95 \%, 87 / 92){ }^{16}$ A recent meta-analysis showed that the SVR rate did not differ between HCC and non-HCC Asian patients with HCV genotype 2 infection who received sofosbuvir in combination with ribavirin $(89.2 \%$ vs. $95.0 \%, P=0.10) .{ }^{17}$ Another meta-analysis that focused on Asian patients with HCV genotype 1 infection also showed that the SVR rate did not differ between HCC and non-HCC patients who received daclatasvir/asunaprevir ( $88.7 \%$ vs. $88.0 \%$, $P=0.98$ ). However, the SVR rate was significantly lower in HCC patients who received ledipasvir/sofosbuvir_tibavirin, compared to those patients without HCC $(94.1 \%$ vs. $98.7 \%, P=0.001) .{ }^{18}$ These findings should be interpreted with the following caveats. Most the studies were retrospective in nature, and some of the studies had a limited number of enrolled HCC cases. Importantly, the HCC status at the time of DAA treatment was not clearly addressed in certain studies. Whether these conclusions were related to the DAA-regimens, disease severity or ethnicities deserves further investigation by more potent DAAs. Recently, a total of $417 \mathrm{HCC}$ patients were enrolled from an international, multicenter cohort in East Asia. Non-HCC controls, who were matched with the HCC patients in regard to age, sex, cirrhosis status, prior treatment experience, HCV genotype, viral loads, platelet counts and biochemistry propensity scores, were enrolled for comparison. The authors concluded that the treatment efficacy did not differ between the patients with and without HCC (95.9\% vs. $94.0 \%$, $P=0.21)^{19}$

\section{CONCLUSIONS}

The definitive conclusion about the treatment efficacy of DAAs in HCC patients remains controversial. The differing results may be attributed to diverse patient characteristics, suboptimal regimens or imprecise definitions of active cancer status at the time of the initiation of treatment. Additional large-scale studies that use the most potent DAAs in clearly defined HCC patients with different disease severities are needed to truly clarify this issue. It has been suggested that all HCV patients should be treated unless they have short life expectancies. Due to the shortage of transplantation resources, it remains unclear as to whether $\mathrm{HCC}$ patients with progressive diseases, in terms of uncontrolled $\mathrm{HCC}$ and poor liver function reserve, should also be aggressively treated, in view of the cost-effectiveness in resource restrained areas. Future studies are also warranted to explore the long-term survival benefits, in terms of overall and cancer-specific mortalities of DAA in patients with active HCC beyond the issue of antiviral efficacy.

\section{Authors' contribution}

CFH and MLY have all contributed to the design and the writing.

\section{Financial support}

The study was supported by grants from Kaohsiung Medical University (MOST 107-2314-B-037-121), Kaohsiung Medical University Hospital (KMUH106-6R05, KMUH106-6M02) and Ministry of Health and Welfare (MOHW106-TDU-B-212-113006 MOHW107-TDU-B-212-123006).

\section{Conflicts of Interest}

The authors have no conflicts to disclose.

\section{REFERENCES}

1. Global Burden of Disease Liver Cancer Collaboration. The burden of primary liver cancer and underlying etiologies from 1990 to 2015 at the global, regional, and national level: results from the global burden of disease study 2015. JAMA Oncol 2017;3:1683-1691.

2. European Association for the Study of the Liver. EASL Clinical Practice Guidelines: management of hepatocellular carcinoma. J Hepatol 2018;69:182-236.

3. Yu ML, Huang CF, Yeh ML, Tsai PC, Huang $\mathrm{Cl}$, Hsieh MH, et al. 
Time-degenerative factors and the risk of hepatocellular carcinoma after antiviral therapy among hepatitis C virus patients: a model for prioritization of treatment. Clin Cancer Res 2017;23:1690-1697.

4. Ioannou GN, Green PK, Berry K. HCV eradication induced by directacting antiviral agents reduces the risk of hepatocellular carcinoma. J Hepatol. 2017 Sep 5. pii: S0168-8278(17)32273-0. doi: 10.1016/ j.jhep.2017.08.030.

5. Huang JF, Yu ML, Huang CF, Chiu CF, Dai CY, Huang Cl, et al. The efficacy and safety of pegylated interferon plus ribavirin combination therapy in chronic hepatitis c patients with hepatocellular carcinoma post curative therapies - a multicenter prospective trial. J Hepatol 2011;54:219-226.

6. Curry MP, Forns X, Chung RT, Terrault NA, Brown R Jr, Fenkel JM, et al. Sofosbuvir and ribavirin prevent recurrence of $\mathrm{HCV}$ infection after liver transplantation: an open-label study. Gastroenterology 2015;148:100-107.e1.

7. Saberi B, Dadabhai AS, Durand CM, Philosophe B, Cameron AM, Sulkowski MS, et al. Challenges in treatment of hepatitis $C$ among patients with hepatocellular carcinoma. Hepatology 2017;66:661-663.

8. Soria A, Fabbiani M, Lapadula G, Gori A. Unexpected viral relapses in hepatitis $C$ virus-infected patients diagnosed with hepatocellular carcinoma during treatment with direct-acting antivirals. Hepatology 2017;66:992-994.

9. Prenner SB, VanWagner LB, Flamm SL, Salem R, Lewandowski RJ, Kulik L. Hepatocellular carcinoma decreases the chance of successful hepatitis C virus therapy with direct-acting antivirals. J Hepatol 2017;66:1173-1181.

10. Beste LA, Green PK, Berry K, Kogut MJ, Allison SK, Ioannou GN. Effectiveness of hepatitis $C$ antiviral treatment in a USA cohort of veteran patients with hepatocellular carcinoma. J Hepatol 2017;67:32-39.

11. Konjeti VR, John BV. Interaction between hepatocellular carcinoma and hepatitis $C$ eradication with direct-acting antiviral therapy. Curr Treat Options Gastroenterol 2018;16:203-214.
12. Furihata T, Matsumoto S, Fu Z, Tsubota A, Sun Y, Matsumoto $S$, et al. Different interaction profiles of direct-acting anti-hepatitis $C$ virus agents with human organic anion transporting polypeptides. Antimicrob Agents Chemother 2014;58:4555-4564.

13. Wlcek K, Svoboda M, Riha J, Zakaria S, Olszewski U, Dvorak Z, et al. The analysis of organic anion transporting polypeptide (OATP) mRNA and protein patterns in primary and metastatic liver cancer. Cancer Biol Ther 2011;11:801-811.

14. Huang CF, Wang SC, Yeh ML, Huang Cl, Tsai PC, Lin ZY, et al. Association of serial serum major histocompatibility complex class I chain-related A measurements with hepatocellular carcinoma in chronic hepatitis C patients after viral eradication. J Gastroenterol Hepatol. 2018 Jun 22. doi: 10.1111/jgh.14359.

15. Sachdeva M, Chawla YK, Arora SK. Immunology of hepatocellular carcinoma. World J Hepatol 2015;7:2080-2090.

16. Chang CY, Nguyen P, Le A, Zhao C, Ahmed A, Daugherty T, et al. Real-world experience with interferon-free, direct acting antiviral therapies in Asian Americans with chronic hepatitis $\mathrm{C}$ and advanced liver disease. Medicine (Baltimore) 2017;96:e6128.

17. Wei B, Ji F, Yeo YH, Ogawa E, Zou B, Stave CD, et al. Real-world effectiveness of sofosbuvir plus ribavirin for chronic hepatitis $C$ genotype 2 in Asia: a systematic review and meta-analysis. BMJ Open Gastroenterol 2018;5:e000207.

18. Ji F, Wei B, Yeo YH, Ogawa E, Zou B, Stave CD, et al. Systematic review with meta-analysis: effectiveness and tolerability of interferonfree direct-acting antiviral regimens for chronic hepatitis $C$ genotype 1 in routine clinical practice in Asia. Aliment Pharmacol Ther 2018:47:550-562.

19. Ogawa $E$, lio $E$, Jun DW, Huang $C F$, Haga $H$, Enomoto $M$, et al. Effectiveness and safety of DAA treatment for $\mathrm{CHC}$ patients with previous HCC: a REAL-C study [Abstract]. American Association for the Study of Liver Diseases (AASLD) 2018:A51. 